УДК 517.98

\author{
S. SHARYN
}

\title{
ALGEBRAIC AND DIFFERENTIAL PROPERTIES OF POLYNOMIAL FOURIER TRANSFORMATION
}

\begin{abstract}
S. Sharyn. Algebraic and differential properties of polynomial Fourier transformation, Mat. Stud. 53 (2020), 59-68.

In the paper, we use the adjoint operator method as well as technique of symmetric tensor products to extended the Fourier transformation onto the spaces of so-called polynomial rapidly decreasing test functions and polynomial tempered distributions. In such spaces it is possible to solve some Cauchy problems, for example, infinite dimensional heat equation associated with the Gross Laplacian.

Algebraic and differential properties of the polynomial Fourier transformation are investigated. We prove some analogical to classical properties of this map. Unlike to the classic case, the spaces of polynomial test and generalized functions have algebraic structure. We prove that polynomial Fourier transformation acts as homomorphism of appropriate algebras. It is clear that the classical analogue of such property is absent.
\end{abstract}

1. Introduction. Integral transformations of test and generalized functions have found a wide range of applications in the theory of differential equations, mathematical physics and other branches of mathematics. Various types of such transforms, their properties and applications have been presented in $[2,11]$.

However, a numerous problems in applied mathematics require a polynomial (nonlinear) generalization of distribution concept. Besides, an algebraic structure of a space of distributions is desirable, which is needed, for example, in quantum field theory [1].

A new approach, that applies the theory of locally convex tensor products together with techniques on symmetric tensor products, is proposed in the papers [5, 9] in order to obtain different polynomial extensions of spaces of ultradifferentiable functions and ultradistributions. In such spaces it is possible to construct a functional calculus for functions of infinity many variables [14] and to solve some Cauchy problems, for example, infinite dimensional heat equation associated with the Gross Laplacian [13].

Note, that there are other known and widely used infinite-dimensional generalizations of classical distribution spaces which are based on modern Gaussian analysis methods as well as the concept of Gelfand triple (see e.g. $[6,8,10]$ ).

In $[5,15]$ the Fourier and Laplace transformations on the space of polynomial ultradistributions are considered, in [15] an appropriate Paley-Wiener-type theorem is proved.

2020 Mathematics Subject Classification: 46F05, 46F12, 46F25, 46E50.

Keywords: polynomials on infinite-dimensional spaces; rapidly decreasing functions; Schwartz distributions; Fourier transformation.

doi:10.30970/ms.53.1.59-68

(C) S. Sharyn, 2020 
In this paper, we extend the Fourier transformation on the spaces of polynomial rapidly decreasing test functions and polynomial tempered distributions and prove some algebraic and differential properties of the transformation.

It is known [16], that the Fourier transformation on the Schwartz space of rapidly decreasing functions (as well as on space of tempered distributions) has the following properties $D^{m} F[f]=F\left[(i t)^{m} f\right]$ and $F\left[D^{m} f\right]=(-i s)^{m} F[f]$, where $D^{m}$ denotes the differentiation operator of the order $m \in \mathbb{Z}_{+}$. In the paper, we prove analogical properties with $m=1$ for (generalized) polynomial Fourier transformation (see Theorems 3, 4 and Corollaries 1, 2). On the other hand, classical Fourier transformation maps a convolution of distributions (if it exists) into a multiplication of respect images, i.e. $F[f * g]=F[f] F[g]$. In Theorem 6 and Corollary 3 we prove the polynomial analogue of this property. The spaces of polynomial test and generalized functions have another algebraic operation, so called Wick product (see formulas (5) and (6)). It is proved, that (generalized) polynomial Fourier transformation acts as homomorphism of appropriate algebras (see Theorem 7 and Corollary 4). It is clear that the classical analogue of this property is absent.

2. Preliminaries and definitions. In what follows $\mathscr{L}(\mathcal{X}, \mathcal{Y})$ denotes the space of all continuous linear operators acting from a locally convex space $\mathcal{X}$ to another such space $\mathcal{Y}$, endowed with the topology of uniform convergence on bounded subsets of $\mathcal{X}$. Let $\mathscr{L}(\mathcal{X}):=$ $\mathscr{L}(\mathcal{X}, \mathcal{X})$. The identity operator in $\mathscr{L}(\mathcal{X})$ always be denoted by $I_{\mathcal{X}}$. The dual space $\mathcal{X}^{\prime}:=$ $\mathscr{L}(\mathcal{X}, \mathbb{C})$ is endowed with strong topology. The pairing between elements of $\mathcal{X}^{\prime}$ and $\mathcal{X}$ we denote $\langle\cdot, \cdot\rangle$.

Let $\mathcal{X}^{\otimes n}$ (resp. $\mathcal{X}^{\widehat{\otimes} n}$ ), $n \in \mathbb{N}$, be the usual (resp. symmetric) $n$th tensor degree of $\mathcal{X}$, completed in the projective tensor topology. For any $x \in \mathcal{X}$ we denote $x^{\otimes n}:=\underbrace{x \otimes \cdots \otimes x}_{n} \in$ $\mathcal{X}^{\widehat{\otimes} n}, n \in \mathbb{N}$. Set $\mathcal{X}^{\widehat{\otimes} 0}:=\mathbb{C}, x^{\otimes 0}:=1 \in \mathbb{C}$.

Let $\mathcal{S}_{p}$ be the Banach space of infinitely differentiable functions on $\mathbb{R}$ with the finite norm

$$
\|\varphi\|_{p}:=\sup _{t \in \mathbb{R}} \sup _{0 \leq m \leq p}\left(1+t^{2}\right)^{p / 2}\left|D^{m} \varphi(t)\right|, \quad p \in \mathbb{Z}_{+}:=\{0\} \cup \mathbb{N},
$$

where $D^{m}$ denotes the differentiation operator of the order $m \in \mathbb{Z}_{+}$. Each inclusion $\mathcal{S}_{p+1} \subset$ $\mathcal{S}_{p}, p \in \mathbb{Z}_{+}$, is compact (see $[16,17]$ ). So the Schwartz space $\mathcal{S}:=\bigcap_{p \in \mathbb{Z}_{+}} \mathcal{S}_{p}$ of all infinitely differentiable rapidly decreasing functions on $\mathbb{R}$ we can endow with the topology of projective $\operatorname{limit} \lim \operatorname{pr}_{p} \mathcal{S}_{p}$ with respect to these inclusions. As a consequence we obtain that $\mathcal{S}$ is Montel nuclear $F S$-space, and its dual space $\mathcal{S}^{\prime}$ of tempered distributions is Montel nuclear $D F S$-space (see [17]). Note that strong topology on $\mathcal{S}^{\prime}$ coincides with Mackey topology and inductive limit topology (see [12, IV.4, IV.5]).

To define the locally convex space $\mathcal{P}_{n}\left(\mathcal{S}^{\prime}\right)$ of all continuous $n$-homogeneous polynomials on $\mathcal{S}^{\prime}$ we use the linear topological isomorphism $\mathcal{P}_{n}\left(\mathcal{S}^{\prime}\right) \simeq\left(\mathcal{S}^{\prime} \widehat{\otimes} n\right)^{\prime}$ described in [4]. Indeed, for any functional $p_{n} \in\left(\mathcal{S}^{\prime \widehat{\otimes} n}\right)^{\prime}$ let us define an $n$-homogeneous polynomial $P_{n} \in \mathcal{P}_{n}\left(\mathcal{S}^{\prime}\right)$ by the formula

$$
P_{n}(f):=\left\langle p_{n}, f^{\otimes n}\right\rangle, \quad f \in \mathcal{S}^{\prime} .
$$

The space $\mathcal{P}_{n}\left(\mathcal{S}^{\prime}\right)$ will be endowed with the locally convex topology $\mathfrak{b}$ of uniform convergence on bounded subsets of $\mathcal{S}^{\prime}$. By definition $\mathcal{P}_{0}\left(\mathcal{S}^{\prime}\right):=\mathbb{C}$. Define the space $\mathcal{P}\left(\mathcal{S}^{\prime}\right)$ of all continuous polynomials on $\mathcal{S}^{\prime}$ as complex linear span of all $\mathcal{P}_{n}\left(\mathcal{S}^{\prime}\right), n \in \mathbb{Z}_{+}$, and endow it with the topology $\mathfrak{b}$. Let $\mathcal{P}^{\prime}\left(\mathcal{S}^{\prime}\right)$ be its strong dual space. In what follows we use the notations

$$
\Gamma(\mathcal{S}):=\bigoplus_{n \in \mathbb{Z}_{+}} \mathcal{S}^{\widehat{\otimes} n} \text { and } \Gamma\left(\mathcal{S}^{\prime}\right):=\underset{n \in \mathbb{Z}_{+}}{\times} \mathcal{S}^{\prime \widehat{\otimes} n}
$$


where $\bigoplus$ and $\times$ denote direct sum and cartesian product respectively. Note that we consider the case when the elements of the direct sum consist of finite but not fixed number of addends.

From the results of the article [9] it follows that there exist the following linear topological isomorphisms

$$
\Upsilon_{n}: \mathcal{P}_{n}\left(\mathcal{S}^{\prime}\right) \longrightarrow \mathcal{S}^{\widehat{\otimes} n}, \quad \Psi_{n}: \mathcal{P}_{n}(\mathcal{S}) \longrightarrow S^{\prime \widehat{\otimes} n}
$$

and

$$
\Upsilon: \mathcal{P}\left(\mathcal{S}^{\prime}\right) \longrightarrow \Gamma(\mathcal{S}), \quad \Psi: \mathcal{P}^{\prime}\left(\mathcal{S}^{\prime}\right) \longrightarrow \Gamma\left(\mathcal{S}^{\prime}\right) \text {. }
$$

Elements of the spaces $\mathcal{P}\left(\mathcal{S}^{\prime}\right)$ and $\mathcal{P}^{\prime}\left(\mathcal{S}^{\prime}\right)$ we call the polynomial rapidly decreasing test functions and polynomial tempered distributions respectively. In what follows elements of the spaces $\Gamma(\mathcal{S})$ and $\Gamma\left(\mathcal{S}^{\prime}\right)$ will be respectively written as

$$
\bigoplus_{n=0}^{m} p_{n}=\left(p_{0}, p_{1}, \ldots, p_{m}, 0, \ldots\right) \text { and } \underset{n \in \mathbb{Z}_{+}}{\times} u_{n}=\left(u_{0}, u_{1}, \ldots, u_{n}, \ldots\right)
$$

for some $m \in \mathbb{N}$, where $p_{k} \in \mathcal{S}^{\widehat{\otimes} k}$ and $u_{k} \in \mathcal{S}^{\prime \widehat{\otimes} k}, k \in \mathbb{Z}_{+}$. To simplify, we write $\left(p_{n}\right)$ and $\left(u_{n}\right)$ instead of $\bigoplus_{n=0}^{m} p_{n}$ and $\times_{n \in \mathbb{Z}_{+}} u_{n}$ respectively.

Note that the following systems of elements

$$
\left\{\left(\varphi^{\otimes n}\right): \varphi \in \mathcal{S}\right\}, \quad\left\{\left(f^{\otimes n}\right): f \in \mathcal{S}^{\prime}\right\}
$$

are total sets in the spaces $\Gamma(\mathcal{S})$ and $\Gamma\left(\mathcal{S}^{\prime}\right)$ respectively.

The spaces $\mathcal{P}\left(\mathcal{S}^{\prime}\right)$ and $\mathcal{P}^{\prime}\left(\mathcal{S}^{\prime}\right)$ are multiplicative algebras with respect to the convolution type operations

$$
P \diamond Q:=\sum_{n \in \mathbb{Z}_{+}} \sum_{m=0}^{n} P_{m} \cdot Q_{n-m} \text { and } U \diamond V:=\underset{n \in \mathbb{Z}_{+}}{\times} \sum_{m=0}^{n} U_{m} \cdot V_{n-m}
$$

respectively, where

$$
\begin{aligned}
& P=\sum_{n \in \mathbb{Z}_{+}} P_{n}, Q=\sum_{n \in \mathbb{Z}_{+}} Q_{n}, P, Q \in \mathcal{P}\left(\mathcal{S}^{\prime}\right), P_{n}, Q_{n} \in \mathcal{P}_{n}\left(\mathcal{S}^{\prime}\right), \\
& U=\underset{n \in \mathbb{Z}_{+}}{\times} U_{n}, V=\underset{n \in \mathbb{Z}_{+}}{\times} V_{n}, U, V \in \mathcal{P}^{\prime}\left(\mathcal{S}^{\prime}\right), U_{n}, V_{n} \in \mathcal{P}_{n}(\mathcal{S}) .
\end{aligned}
$$

Note, that in the above formulas $P_{m} \cdot Q_{n-m}$ and $U_{m} \cdot V_{n-m}$ denote the usual pointwise multiplication of polynomials.

The direct sum $\Gamma(\mathcal{S})$ and the cartesian product $\Gamma\left(\mathcal{S}^{\prime}\right)$ are local convex algebras with respect to the convolution type operations

$$
p \diamond q:=\bigoplus_{n \in \mathbb{Z}_{+}} \sum_{m=0}^{n} p_{m} \widehat{\otimes} q_{n-m} \quad \text { and } \quad u \diamond v:=\underset{n \in \mathbb{Z}_{+}}{\times} \sum_{m=0}^{n} u_{m} \widehat{\otimes} v_{n-m}
$$

respectively, where $p=\left(p_{n}\right), q=\left(q_{n}\right), p, q \in \Gamma(\mathcal{S}), p_{n}, q_{n} \in \mathcal{S}^{\widehat{\otimes} n}, u=\left(u_{n}\right), v=\left(v_{n}\right)$, $u, v \in \Gamma\left(\mathcal{S}^{\prime}\right), u_{n}, v_{n} \in \mathcal{S}^{\widehat{\otimes} \otimes n}$.

Note that, the convolution type operations (5), (6) play an inportant role in stochastic analysis, they are called the Wick product in the literature (see, e.g., $[3,7]$ ). 
The mappings (3) act as algebraic isomorphisms, i.e.

$$
\Upsilon:\left\{\mathcal{P}\left(\mathcal{S}^{\prime}\right), \diamond\right\} \longrightarrow\{\Gamma(\mathcal{S}), \diamond\}, \quad \Psi:\left\{\mathcal{P}^{\prime}\left(\mathcal{S}^{\prime}\right), \diamond\right\} \longrightarrow\left\{\Gamma\left(\mathcal{S}^{\prime}\right), \diamond\right\} .
$$

3. Polynomial generalization of the Fourier transform. As is well known the usual Fourier transformation

$$
F: \mathcal{S} \in \varphi(t) \longmapsto[F \varphi](\xi):=\frac{1}{\sqrt{2 \pi}} \int_{\mathbb{R}} e^{-i t \xi} \varphi(t) d t \in \mathcal{S}
$$

acts as continuous bijection from the space $\mathcal{S}$ onto itself. Let $F^{\prime} \in \mathscr{L}\left(\mathcal{S}^{\prime}\right)$ be the generalized Fourier transformation on the space $\mathcal{S}^{\prime}$, i.e. the map defined by the formula $\left\langle F^{\prime} f, \varphi\right\rangle=$ $\langle f, F \varphi\rangle, f \in \mathcal{S}^{\prime}, \varphi \in \mathcal{S}$.

For each natural $n$ we define the operators $F^{\otimes n} \in \mathscr{L}\left(\mathcal{S}^{\otimes} n\right)$ and $F^{\prime \otimes n} \in \mathscr{L}\left(\mathcal{S}^{\prime \otimes n}\right)$ as linear and continuous extensions of mappings

$$
\begin{aligned}
\varphi_{1} \widehat{\otimes} \ldots \widehat{\otimes} \varphi_{n} & \longmapsto \quad F \varphi_{1} \widehat{\otimes} \ldots \widehat{\otimes} F \varphi_{n}, \quad \varphi_{i} \in \mathcal{S}, \quad i=1, \ldots, n, \\
f_{1} \widehat{\otimes} \ldots \widehat{\otimes} f_{n} & \longmapsto \quad F^{\prime} f_{1} \widehat{\otimes} \ldots \widehat{\otimes} F^{\prime} f_{n}, \quad f_{i} \in \mathcal{S}^{\prime}, \quad i=1, \ldots, n,
\end{aligned}
$$

respectively, and let $F^{\otimes 0}:=F^{\otimes \otimes 0}:=I_{\mathbb{C}}$ by definition.

We define the operators $F^{\otimes} \in \mathscr{L}(\Gamma(\mathcal{S}))$ and $F^{\prime \otimes} \in \mathscr{L}\left(\Gamma\left(\mathcal{S}^{\prime}\right)\right)$ as follows:

$$
\begin{aligned}
& F^{\otimes}:=\underset{n \in \mathbb{Z}_{+}}{\times} F^{\otimes n}: \quad \Gamma(\mathcal{S}) \ni p=\left(p_{n}\right) \quad \longmapsto \quad F^{\otimes} p:=\left(F^{\otimes n} p_{n}\right) \in \Gamma(\mathcal{S}), \\
& F^{\prime \otimes}:=\underset{n \in \mathbb{Z}_{+}}{\times} F^{\prime \otimes n}: \quad \Gamma\left(\mathcal{S}^{\prime}\right) \ni u=\left(u_{n}\right) \longmapsto F^{\prime \otimes} u:=\left(F^{\prime \otimes n} u_{n}\right) \in \Gamma\left(\mathcal{S}^{\prime}\right),
\end{aligned}
$$

where $p_{n} \in \mathcal{S}^{\widehat{\otimes} n}, u_{n} \in \mathcal{S}^{\prime \widehat{\otimes} n}$.

Theorem 1. The following diagrams
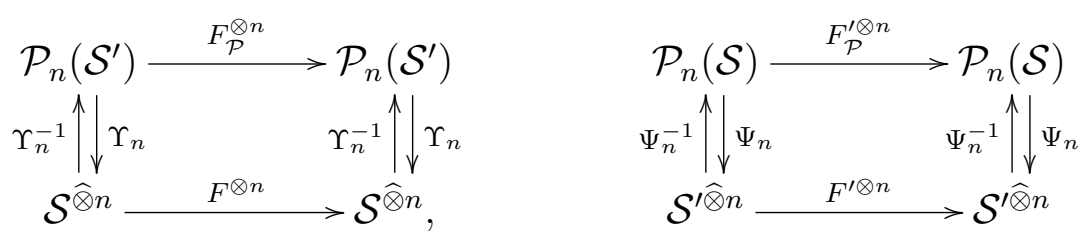

uniquely define linear continuous operators $F_{\mathcal{P}}^{\otimes n} \in \mathscr{L}\left(\mathcal{P}_{n}\left(\mathcal{S}^{\prime}\right)\right)$ and $F_{\mathcal{P}}^{\prime \otimes n} \in \mathscr{L}\left(\mathcal{P}_{n}(\mathcal{S})\right)$, which are adjoint to each other.

Proof. Isomorphisms (2) imply that above diagrams are commutative. So, linear mappings $F_{\mathcal{P}}^{\otimes n}:=\Upsilon_{n}^{-1} \circ F^{\otimes n} \circ \Upsilon_{n}$ and $F_{\mathcal{P}}^{\prime \otimes n}:=\Psi_{n}^{-1} \circ F^{\prime \otimes n} \circ \Psi_{n}$ uniquely can be defined by the following equalities (see formula (1))

$$
\begin{aligned}
& {\left[F_{\mathcal{P}}^{\otimes n} P_{n}\right](f):=\left\langle p_{n}, F^{\otimes n} f^{\otimes n}\right\rangle, \quad f \in \mathcal{S}^{\prime}, \quad P_{n} \in \mathcal{P}_{n}\left(\mathcal{S}^{\prime}\right),} \\
& {\left[F_{\mathcal{P}}^{\prime \otimes n} U_{n}\right](\varphi):=\left\langle u_{n}, F^{\otimes n} \varphi^{\otimes n}\right\rangle, \quad \varphi \in \mathcal{S}, \quad U_{n} \in \mathcal{P}_{n}(\mathcal{S}),}
\end{aligned}
$$

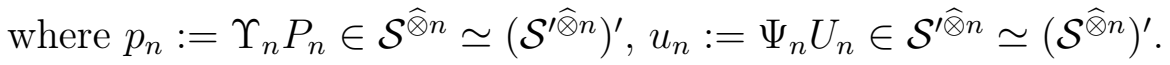

What is left to show that the operators $F^{\otimes n}$ and $F^{\prime \otimes n}$ are continuous. Note, that these operators are mutually adjoint, i.e.

$$
\left\langle F^{\prime} f_{1} \widehat{\otimes} \ldots \widehat{\otimes} F^{\prime} f_{n}, \varphi_{1} \widehat{\otimes} \ldots \widehat{\otimes} \varphi_{n}\right\rangle=\left\langle f_{1} \widehat{\otimes} \ldots \widehat{\otimes} f_{n}, F \varphi_{1} \widehat{\otimes} \ldots \widehat{\otimes} F \varphi_{n}\right\rangle,
$$


where $f_{1} \widehat{\otimes} \ldots \widehat{\otimes} f_{n} \in \mathcal{S}^{\prime \widehat{\otimes} n}, \varphi_{1} \widehat{\otimes} \ldots \widehat{\otimes} \varphi_{n} \in \mathcal{S}^{\widehat{\otimes} n}, f_{i} \in \mathcal{S}^{\prime}, \varphi_{i} \in \mathcal{S}, i=1, \ldots, n$.

The continuity of the Fourier transformation on $\mathcal{S}$ is well known (see [16]): for any $m \in \mathbb{Z}_{+}$ there exists a constant $C_{m}$ such that $\|F \varphi\|_{m} \leq C_{m}\|\varphi\|_{m+2}$. So, for any continuous seminorm $q_{1} \otimes \ldots \otimes q_{n}$ on $\mathcal{S}^{\otimes n}$ there exist constants $C_{i_{1}}, \ldots, C_{i_{n}}$ and indexes $m_{i_{1}}, \ldots, m_{i_{n}}, i=1, \ldots, k$, such that

$$
\begin{gathered}
\left(q_{1} \otimes \ldots \otimes q_{n}\right)\left(F^{\otimes n} \boldsymbol{\varphi}\right)=\inf \sum_{i=1}^{k}\left\|F \varphi_{i_{1}}\right\|_{m_{i_{1}}} \cdots\left\|F \varphi_{i_{n}}\right\|_{m_{i_{n}}} \leq \\
\leq \inf \sum_{i=1}^{k} C_{i_{1}}\left\|\varphi_{i_{1}}\right\|_{m_{i_{1}}+2} \cdots C_{i_{n}}\left\|\varphi_{i_{n}}\right\|_{m_{i_{n}}+2}=C\left(p_{1} \otimes \ldots \otimes p_{n}\right)(\boldsymbol{\varphi}),
\end{gathered}
$$

where the infimum is taken over all representations of an element $\varphi \in \mathcal{S}^{\otimes n}$ in the form $\boldsymbol{\varphi}=\sum_{i=1}^{k} \varphi_{i_{1}} \otimes \cdots \otimes \varphi_{i_{n}}, \varphi_{i_{j}} \in \mathcal{S}, i=1, \ldots, k, j=1, \ldots, n$. It implies the continuity of the operator $F^{\otimes n}: \mathcal{S}^{\otimes n} \longrightarrow \mathcal{S}^{\otimes n}$.

The symmetrization projector

$$
\mathfrak{s}_{n}: \mathcal{S}^{\otimes n} \ni \varphi_{1} \otimes \cdots \otimes \varphi_{n} \longmapsto \varphi_{1} \widehat{\otimes} \cdots \widehat{\otimes} \varphi_{n}:=\frac{1}{n !} \sum_{\sigma} \varphi_{\sigma(1)} \otimes \cdots \otimes \varphi_{\sigma(n)},
$$

where the sum is taken over all permutations $\sigma$ of the set $\{1,2, \ldots, n\}$, is continuous. It easy to see, that $\mathfrak{s}_{n} \circ F^{\otimes n}=F^{\otimes n} \circ \mathfrak{s}_{n}$, since the set of symmetric tensors is invariant with respect to the action of the operator $F^{\otimes n}$. Hence, the restriction $F^{\otimes n}: \mathcal{S}^{\widehat{\otimes} n} \longrightarrow \mathcal{S}^{\widehat{\otimes} n}$ is continuous.

The proof of continuity of the adjoint operator $F^{\prime \otimes n}: \mathcal{S}^{\prime \widehat{\otimes} n} \longrightarrow \mathcal{S}^{\prime \widehat{\otimes} n}$ is similar.

Theorem 2. The following diagrams
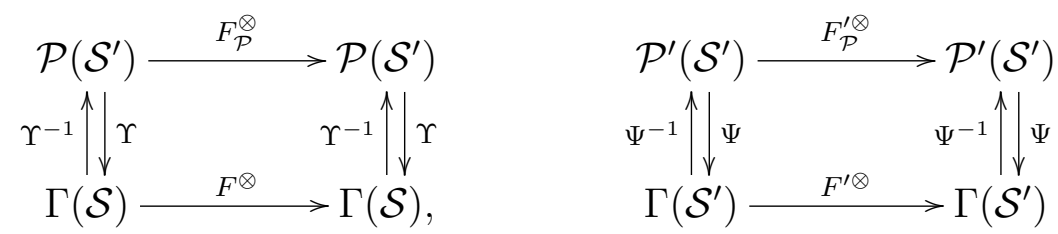

uniquely define linear continuous operators $F_{\mathcal{P}}^{\otimes} \in \mathscr{L}\left(\mathcal{P}\left(\mathcal{S}^{\prime}\right)\right), F_{\mathcal{P}}^{\prime \otimes} \in \mathscr{L}\left(\mathcal{P}^{\prime}\left(\mathcal{S}^{\prime}\right)\right)$, which are adjoint to each other.

Proof. Isomorphisms (3) imply that above diagrams are commutative. So, linear mappings $F_{\mathcal{P}}^{\otimes}:=\Upsilon^{-1} \circ F^{\otimes} \circ \Upsilon$ and $F_{\mathcal{P}}^{\prime \otimes}:=\Psi^{-1} \circ F^{\prime \otimes} \circ \Psi$ uniquely can be defined by the following equalities

$$
F_{\mathcal{P}}^{\otimes} P:=\sum_{n \in \mathbb{Z}_{+}} F_{\mathcal{P}}^{\otimes n} P_{n}, \quad F_{\mathcal{P}}^{\prime \otimes} U:=\underset{n \in \mathbb{Z}_{+}}{\times} F_{\mathcal{P}}^{\prime \otimes n} U_{n}
$$

where

$$
P=\sum_{n \in \mathbb{Z}_{+}} P_{n} \in \mathcal{P}\left(\mathcal{S}^{\prime}\right), P_{n} \in \mathcal{P}_{n}\left(\mathcal{S}^{\prime}\right), U=\underset{n \in \mathbb{Z}_{+}}{\times} U_{n} \in \mathcal{P}^{\prime}\left(\mathcal{S}^{\prime}\right), U_{n} \in \mathcal{P}_{n}(\mathcal{S})
$$

Theorem 1 implies the following equalities

$$
\begin{aligned}
& \left\langle U, F_{\mathcal{P}}^{\otimes} P\right\rangle=\left\langle\underset{n \in \mathbb{Z}_{+}}{\times} U_{n}, \sum_{n \in \mathbb{Z}_{+}} F_{\mathcal{P}}^{\otimes n} P_{n}\right\rangle=\sum_{n \in \mathbb{Z}_{+}}\left\langle U_{n}, F_{\mathcal{P}}^{\otimes n} P_{n}\right\rangle= \\
& =\sum_{n \in \mathbb{Z}_{+}}\left\langle F_{\mathcal{P}}^{\prime \otimes n} U_{n}, P_{n}\right\rangle=\left\langle\underset{n \in \mathbb{Z}_{+}}{\times} F_{\mathcal{P}}^{\prime \otimes n} U_{n}, \sum_{n \in \mathbb{Z}_{+}} P_{n}\right\rangle=\left\langle F_{\mathcal{P}}^{\prime \otimes} U, P\right\rangle,
\end{aligned}
$$


therefore the operators $F_{\mathcal{P}}^{\otimes}$ and $F_{\mathcal{P}}^{\prime \otimes}$ are mutually adjoint.

In the proof of the Theorem 1 we have shown continuity of the operators $F_{\mathcal{P}}^{\otimes n}$ and $F_{\mathcal{P}}^{\prime \otimes n}$. Hence, from the definitions of locally convex topologies of direct sum and cartesian product it follows the continuity of the operators $F_{\mathcal{P}}^{\otimes} \in \mathscr{L}\left(\mathcal{P}\left(\mathcal{S}^{\prime}\right)\right)$ and $F_{\mathcal{P}}^{\prime \otimes} \in \mathscr{L}\left(\mathcal{P}^{\prime}\left(\mathcal{S}^{\prime}\right)\right)$.

The mapping $F_{\mathcal{P}}^{\otimes} \in \mathscr{L}\left(\mathcal{P}\left(\mathcal{S}^{\prime}\right)\right.$ ) (respectively $F_{\mathcal{P}}^{\prime \otimes} \in \mathscr{L}\left(\mathcal{P}^{\prime}\left(\mathcal{S}^{\prime}\right)\right)$ ), defined in Theorem 2, we will call (respectively generalized) polynomial Fourier transformation.

4. Auxilary operations. Let $D^{\prime} \in \mathscr{L}\left(\mathcal{S}^{\prime}\right)$ be the operator of generalized differentiation, i.e. the map defined by the formula $\left\langle D^{\prime} f, \varphi\right\rangle=-\langle f, D \varphi\rangle, f \in \mathcal{S}^{\prime}, \varphi \in \mathcal{S}$, where $D$ denotes the operator of usual differentiation in $\mathcal{S}$. Let us extend the operators $D \in \mathscr{L}(\mathcal{S})$ and $D^{\prime} \in \mathscr{L}\left(\mathcal{S}^{\prime}\right)$ onto the spaces $\Gamma(\mathcal{S})$ and $\Gamma\left(\mathcal{S}^{\prime}\right)$ respectively. Namely, define the operators $\mathbb{D} \in \mathscr{L}(\Gamma(\mathcal{S}))$ and $\mathbb{D}^{\prime} \in \mathscr{L}\left(\Gamma\left(\mathcal{S}^{\prime}\right)\right)$ by the following formulas

$$
\begin{aligned}
\mathbb{D} p:=\bigoplus_{n \in \mathbb{Z}_{+}} D^{\{\otimes\} n} \varphi^{\otimes n}, \quad p=\left(\varphi^{\otimes n}\right) \in \Gamma(\mathcal{S}), \quad \varphi \in \mathcal{S}, \\
\mathbb{D}^{\prime} u:=\underset{n \in \mathbb{Z}_{+}}{\times} D^{\prime\{\otimes\} n} f^{\otimes n}, \quad u=\left(f^{\otimes n}\right) \in \Gamma\left(\mathcal{S}^{\prime}\right), \quad f \in \mathcal{S}^{\prime},
\end{aligned}
$$

where $D^{\{\otimes\} 0}$ and $D^{\prime\{\otimes\} 0}$ are null operators and

$$
\begin{array}{ll}
D^{\{\otimes\} n} \varphi^{\otimes n}:=\sum_{j=1}^{n} \varphi^{\otimes(j-1)} \otimes D \varphi \otimes \varphi^{\otimes(n-j)}, & n \in \mathbb{N} \\
D^{\prime\{\otimes\} n} f^{\otimes n}:=\sum_{j=1}^{n} f^{\otimes(j-1)} \otimes D^{\prime} f \otimes f^{\otimes(n-j)}, & n \in \mathbb{N} .
\end{array}
$$

Let us define the operator $M \in \mathscr{L}(\mathcal{S})$ of multiplication on the independent variable by $M: \mathcal{S} \ni \varphi(t) \longmapsto-i t \varphi(t) \in \mathcal{S}$ and let $M^{\prime} \in \mathscr{L}\left(\mathcal{S}^{\prime}\right)$ be its adjoint map, i.e. $\left\langle M^{\prime} f, \varphi\right\rangle=$ $\langle f, M \varphi\rangle, f \in \mathcal{S}^{\prime}, \varphi \in \mathcal{S}$. Next we extend these operators onto the spaces $\Gamma(\mathcal{S})$ and $\Gamma\left(\mathcal{S}^{\prime}\right)$ in analogous way. Namely, define the operators $\mathbb{M} \in \mathscr{L}(\Gamma(\mathcal{S}))$ and $\mathbb{M}^{\prime} \in \mathscr{L}\left(\Gamma\left(\mathcal{S}^{\prime}\right)\right)$ by

$$
\begin{gathered}
\mathbb{M} p:=\bigoplus_{n \in \mathbb{Z}_{+}} M^{\{\otimes\} n} \varphi^{\otimes n}, \quad p=\left(\varphi^{\otimes n}\right) \in \Gamma(\mathcal{S}), \quad \varphi \in \mathcal{S}, \\
\mathbb{M}^{\prime} u:=\underset{n \in \mathbb{Z}_{+}}{\times} M^{\prime\{\otimes\} n} f^{\otimes n}, \quad u=\left(f^{\otimes n}\right) \in \Gamma\left(\mathcal{S}^{\prime}\right), \quad f \in \mathcal{S}^{\prime},
\end{gathered}
$$

where $M^{\{\otimes\} 0}$ and $M^{\prime\{\otimes\} 0}$ are null operators and

$$
\begin{aligned}
M^{\{\otimes\} n} \varphi^{\otimes n}:=\sum_{j=1}^{n} \varphi^{\otimes(j-1)} \otimes M \varphi \otimes \varphi^{\otimes(n-j)}, & n \in \mathbb{N} \\
M^{\prime\{\otimes\} n} f^{\otimes n}:=\sum_{j=1}^{n} f^{\otimes(j-1)} \otimes M^{\prime} f \otimes f^{\otimes(n-j)}, & n \in \mathbb{N} .
\end{aligned}
$$

The following commutative diagrams
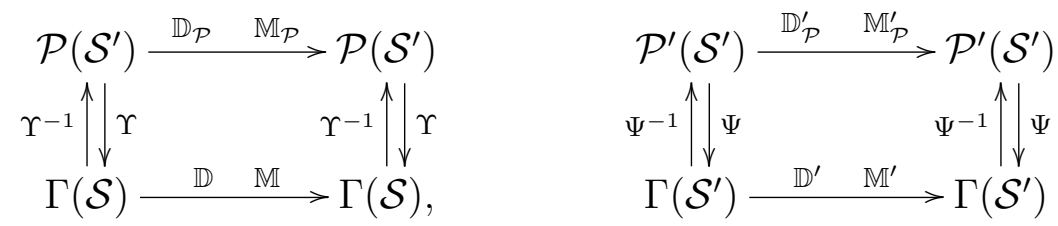
uniquely define linear continuous operators $\mathbb{D}_{\mathcal{P}}:=\Upsilon^{-1} \circ \mathbb{D} \circ \Upsilon \in \mathscr{L}\left(\mathcal{P}\left(\mathcal{S}^{\prime}\right)\right), \mathbb{M}_{\mathcal{P}}:=\Upsilon^{-1} \circ$ $\mathbb{M} \circ \Upsilon \in \mathscr{L}\left(\mathcal{P}\left(\mathcal{S}^{\prime}\right)\right), \mathbb{D}_{\mathcal{P}}^{\prime}:=\Psi^{-1} \circ \mathbb{D}^{\prime} \circ \Psi \in \mathscr{L}\left(\mathcal{P}^{\prime}\left(\mathcal{S}^{\prime}\right)\right)$ and $\mathbb{M}_{\mathcal{P}}^{\prime}:=\Psi^{-1} \circ \mathbb{M}^{\prime} \circ \Psi \in \mathscr{L}\left(\mathcal{P}^{\prime}\left(\mathcal{S}^{\prime}\right)\right)$.

\section{Differential properties.}

Theorem 3. For any elements $p \in \Gamma(\mathcal{S})$ and $u \in \Gamma\left(\mathcal{S}^{\prime}\right)$ the following equalities are valid

$$
\mathbb{D}\left[F^{\otimes} p\right]=F^{\otimes}[\mathbb{M} p], \quad \mathbb{D}^{\prime}\left[F^{\prime \otimes} u\right]=F^{\prime \otimes}\left[\mathbb{M}^{\prime} u\right] .
$$

Proof. It easy to see, that for any $\varphi \in \mathcal{S}$ we have

$$
\begin{gathered}
D[F \varphi](\xi)=\frac{1}{\sqrt{2 \pi}} \int_{\mathbb{R}}(-\mathfrak{i} t) e^{-i t \xi} \varphi(t) d t=F[M \varphi] \\
F[D \varphi](\xi)=\frac{1}{\sqrt{2 \pi}} \int_{\mathbb{R}} e^{-\mathfrak{i} t \xi} \varphi^{\prime}(t) d t=\frac{\mathfrak{i} \xi}{\sqrt{2 \pi}} \int_{\mathbb{R}} e^{-\mathfrak{i} t \xi} \varphi(t) d t=-M[F \varphi] .
\end{gathered}
$$

It follows

$$
\left\langle D^{\prime}\left[F^{\prime} f\right], \varphi\right\rangle=-\left\langle F^{\prime} f, D \varphi\right\rangle=-\langle f, F[D \varphi]\rangle=\langle f, M[F \varphi]\rangle=\left\langle M^{\prime} f, F \varphi\right\rangle=\left\langle F^{\prime}\left[M^{\prime} f\right], \varphi\right\rangle,
$$

so, $D^{\prime}\left[F^{\prime} f\right]=F^{\prime}\left[M^{\prime} f\right]$.

It is clear, that we only need to check the equalities (8) on total subsets (4) in $\Gamma(\mathcal{S})$ and $\Gamma\left(\mathcal{S}^{\prime}\right)$. Let $p=\left(\varphi^{\otimes n}\right)$ and $u=\left(f^{\otimes n}\right)$ with $\varphi \in \mathcal{S}$ and $f \in \mathcal{S}^{\prime}$.

Then we have

$$
\begin{gathered}
\mathbb{D}\left[F^{\otimes} p\right]=\mathbb{D}\left[\bigoplus_{n \in \mathbb{Z}_{+}} F^{\otimes n} \varphi^{\otimes n}\right]=\mathbb{D}\left[\bigoplus_{n \in \mathbb{Z}_{+}}(F \varphi)^{\otimes n}\right]=\bigoplus_{n \in \mathbb{Z}_{+}} D^{\{\otimes\} n}(F \varphi)^{\otimes n}= \\
=0 \oplus \bigoplus_{n \in \mathbb{N}} \sum_{j=1}^{n}(F \varphi)^{\otimes(j-1)} \otimes D[F \varphi] \otimes(F \varphi)^{\otimes(n-j)}= \\
=0 \oplus \bigoplus_{n \in \mathbb{N}} \sum_{j=1}^{n}(F \varphi)^{\otimes(j-1)} \otimes F[M \varphi] \otimes(F \varphi)^{\otimes(n-j)}= \\
=0 \oplus \bigoplus_{n \in \mathbb{N}} F^{\otimes n} \sum_{j=1}^{n} \varphi^{\otimes(j-1)} \otimes M \varphi \otimes \varphi^{\otimes(n-j)}=0 \oplus \bigoplus_{n \in \mathbb{N}} F^{\otimes n} M^{\{\otimes\} n} \varphi^{\otimes n}=F^{\otimes}[\mathbb{M} p]
\end{gathered}
$$

and

$$
\begin{gathered}
\mathbb{D}^{\prime}\left[F^{\prime \otimes} u\right]=\mathbb{D}^{\prime}\left[\underset{n \in \mathbb{Z}_{+}}{\times} F^{\otimes n} f^{\otimes n}\right]=\mathbb{D}^{\prime}\left[\underset{n \in \mathbb{Z}_{+}}{\times}\left(F^{\prime} f\right)^{\otimes n}\right]=\underset{n \in \mathbb{Z}_{+}}{\times} D^{\prime\{\otimes\} n}\left(F^{\prime} f\right)^{\otimes n} \\
=0 \times \underset{n \in \mathbb{N}}{\times} \sum_{j=1}^{n}\left(F^{\prime} f\right)^{\otimes(j-1)} \otimes D^{\prime}\left[F^{\prime} f\right] \otimes\left(F^{\prime} f\right)^{\otimes(n-j)} \\
=0 \times \underset{n \in \mathbb{N}}{\times} \sum_{j=1}^{n}\left(F^{\prime} f\right)^{\otimes(j-1)} \otimes F^{\prime}\left[M^{\prime} f\right] \otimes\left(F^{\prime} f\right)^{\otimes(n-j)} \\
=0 \times \underset{n \in \mathbb{N}}{\times} F^{\prime \otimes n} \sum_{j=1}^{n} f^{\otimes(j-1)} \otimes M^{\prime} f \otimes f^{\otimes(n-j)}=0 \times \underset{n \in \mathbb{N}}{\times} F^{\otimes n} M^{\prime\{\otimes\} n} f^{\otimes n}=F^{\prime \otimes}\left[\mathbb{M}^{\prime} u\right] .
\end{gathered}
$$


Corollary 1. For any elements $P \in \mathcal{P}\left(\mathcal{S}^{\prime}\right)$ and $U \in \mathcal{P}^{\prime}\left(\mathcal{S}^{\prime}\right)$ the following equalities are valid

$$
\mathbb{D}_{\mathcal{P}}\left[F_{\mathcal{P}}^{\otimes} P\right]=F_{\mathcal{P}}^{\otimes}\left[\mathbb{M}_{\mathcal{P}} P\right], \quad \mathbb{D}_{\mathcal{P}}^{\prime}\left[F_{\mathcal{P}}^{\prime \otimes} U\right]=F_{\mathcal{P}}^{\prime \otimes}\left[\mathbb{M}_{\mathcal{P}}^{\prime} U\right]
$$

Theorem 4. For any elements $p \in \Gamma(\mathcal{S})$ and $u \in \Gamma\left(\mathcal{S}^{\prime}\right)$ the following equalities are valid

$$
F^{\otimes}[\mathbb{D} p]=-\mathbb{M}\left[F^{\otimes} p\right], \quad F^{\prime \otimes}\left[\mathbb{D}^{\prime} u\right]=-\mathbb{M}^{\prime}\left[F^{\prime \otimes} u\right] .
$$

Proof. For any elements $p=\left(\varphi^{\otimes n}\right)$ and $u=\left(f^{\otimes n}\right)$ with $\varphi \in \mathcal{S}$ and $f \in \mathcal{S}^{\prime}$ from the respective total subsets (4) we have

$$
\begin{gathered}
F^{\otimes}[\mathbb{D} p]=F^{\otimes}\left[0 \oplus \bigoplus_{n \in \mathbb{N}} D^{\{\otimes\} n} \varphi^{\otimes n}\right]=0 \oplus \bigoplus_{n \in \mathbb{N}} F^{\otimes n} D^{\{\otimes\} n} \varphi^{\otimes n}= \\
=0 \oplus \bigoplus_{n \in \mathbb{N}} F^{\otimes n} \sum_{j=1}^{n} \varphi^{\otimes(j-1)} \otimes D \varphi \otimes \varphi^{\otimes(n-j)}= \\
=0 \oplus \bigoplus_{n \in \mathbb{N}} \sum_{j=1}^{n}(F \varphi)^{\otimes(j-1)} \otimes F[D \varphi] \otimes(F \varphi)^{\otimes(n-j)}= \\
=-0 \oplus \bigoplus_{n \in \mathbb{N}} \sum_{j=1}^{n}(F \varphi)^{\otimes(j-1)} \otimes M[F \varphi] \otimes(F \varphi)^{\otimes(n-j)}=-0 \oplus \bigoplus_{n \in \mathbb{N}} M^{\{\otimes\} n}(F \varphi)^{\otimes n}=-\mathbb{M}\left[F^{\otimes} p\right] .
\end{gathered}
$$

It easy to check that for any $f \in \mathcal{S}^{\prime}$ and $\varphi \in \mathcal{S}$ the following is true

$$
\left\langle F^{\prime}\left[D^{\prime} f\right], \varphi\right\rangle=\left\langle D^{\prime} f, F \varphi\right\rangle=-\langle f, D[F \varphi]\rangle=-\langle f, F[M \varphi]\rangle=-\left\langle F^{\prime} f, M \varphi\right\rangle=-\left\langle M^{\prime}\left[F^{\prime} f\right], \varphi\right\rangle .
$$

It implies

$$
\begin{gathered}
F^{\otimes}\left[\mathbb{D}^{\prime} u\right]=F^{\prime \otimes}\left[0 \times \underset{n \in \mathbb{N}}{\times} D^{\prime\{\otimes\} n} f^{\otimes n}\right]=0 \times \underset{n \in \mathbb{N}}{\times} F^{\prime \otimes n} D^{\prime\{\otimes\} n} f^{\otimes n}= \\
=0 \times \underset{n \in \mathbb{N}}{\times} F^{\prime \otimes n} \sum_{j=1}^{n} f^{\otimes(j-1)} \otimes D^{\prime} f \otimes f^{\otimes(n-j)}= \\
=0 \times \underset{n \in \mathbb{N}}{\times} \sum_{j=1}^{n}\left(F^{\prime} f\right)^{\otimes(j-1)} \otimes F^{\prime}\left[D^{\prime} f\right] \otimes\left(F^{\prime} f\right)^{\otimes(n-j)}= \\
=-0 \times \underset{n \in \mathbb{N}}{\times} \sum_{j=1}^{n}\left(F^{\prime} f\right)^{\otimes(j-1)} \otimes M^{\prime}\left[F^{\prime} f\right] \otimes\left(F^{\prime} f\right)^{\otimes(n-j)}= \\
=-0 \times \underset{n \in \mathbb{N}}{\times} M^{\prime\{\otimes\} n} F^{\otimes n} f^{\otimes n}=-\mathbb{M}^{\prime}\left[F^{\prime \otimes} u\right] .
\end{gathered}
$$

Corollary 2. For any elements $P \in \mathcal{P}\left(\mathcal{S}^{\prime}\right)$ and $U \in \mathcal{P}^{\prime}\left(\mathcal{S}^{\prime}\right)$ the following equalities are valid

$$
F_{\mathcal{P}}^{\otimes}\left[\mathbb{D}_{\mathcal{P}} P\right]=-\mathbb{M}_{\mathcal{P}}\left[F_{\mathcal{P}}^{\otimes} P\right], \quad F_{\mathcal{P}}^{\prime \otimes}\left[\mathbb{D}_{\mathcal{P}}^{\prime} U\right]=-\mathbb{M}_{\mathcal{P}}^{\prime}\left[F_{\mathcal{P}}^{\prime \otimes} U\right] .
$$

6. Algebraic properties. Let $\mathcal{E}^{\prime} \subset \mathcal{S}^{\prime}$ be the space of generalized functions with compact supports. Denote $\Gamma\left(\mathcal{E}^{\prime}\right):=\times_{n \in \mathbb{Z}_{+}} \mathcal{E}^{\prime \widehat{\otimes} n}$. It is clear that $\Gamma\left(\mathcal{E}^{\prime}\right) \subset \Gamma\left(\mathcal{S}^{\prime}\right)$.

In [16] it is proved the following assertion. 
Theorem 5 ([16]). Let $f \in \mathcal{S}^{\prime}$ and $g \in \mathcal{E}^{\prime}$. Then $f * g \in \mathcal{S}^{\prime}$ is well defined, moreover $F^{\prime}[f * g]=F^{\prime}[f] \cdot F^{\prime}[g]$.

Let us generalize this property onto the space $\Gamma\left(\mathcal{S}^{\prime}\right)$.

For elements $\left(f^{\otimes n}\right),\left(g^{\otimes n}\right), f, g \in \mathcal{S}^{\prime}$, from total subset of $\Gamma\left(\mathcal{S}^{\prime}\right)$ we define two operations

$$
\left(f^{\otimes n}\right) \circledast\left(g^{\otimes n}\right):=\left((f * g)^{\otimes n}\right) \quad \text { and } \quad\left(f^{\otimes n}\right) \odot\left(g^{\otimes n}\right):=\left((f \cdot g)^{\otimes n}\right)
$$

and extend them on $\Gamma\left(\mathcal{S}^{\prime}\right)$ by linearity and continuity. Note, that these operations are not well defined on whole space $\Gamma\left(\mathcal{S}^{\prime}\right)$. But the following result is true, it is a consequence of the Theorem 5 .

Theorem 6. Let $u \in \Gamma\left(\mathcal{S}^{\prime}\right)$ and $v \in \Gamma\left(\mathcal{E}^{\prime}\right)$. Then $u \circledast v \in \Gamma\left(\mathcal{S}^{\prime}\right)$ is well defined, moreover

$$
F^{\prime \otimes}[u \circledast v]=F^{\prime \otimes}[u] \odot F^{\prime \otimes}[v] .
$$

Proof. It is enough to prove the assertion only on the elements of total subsets (4). Let $u=\left(f^{\otimes n}\right) \in \Gamma\left(\mathcal{S}^{\prime}\right), v=\left(g^{\otimes n}\right) \in \Gamma\left(\mathcal{E}^{\prime}\right)$, where $f \in \mathcal{S}^{\prime}, g \in \mathcal{E}^{\prime}$. Then

$F^{\prime \otimes}[u \circledast v]=\underset{n \in \mathbb{Z}_{+}}{\times} F^{\prime \otimes n}(f * g)^{\otimes n}=\underset{n \in \mathbb{Z}_{+}}{\times}\left(F^{\prime}[f * g]\right)^{\otimes n}=\underset{n \in \mathbb{Z}_{+}}{\times}\left(F^{\prime}[f] \cdot F^{\prime}[g]\right)^{\otimes n}=F^{\otimes}[u] \odot F^{\prime \otimes}[v]$.

Using the second of the diagrams $(7)$ we can "extend" the operations $\circledast$ and $\odot$ onto the space $\mathcal{P}^{\prime}\left(\mathcal{S}^{\prime}\right)$ of polynomial distributions.

Corollary 3. Let $U \in \mathcal{P}^{\prime}\left(\mathcal{S}^{\prime}\right)$ and $V \in \mathcal{P}^{\prime}\left(\mathcal{E}^{\prime}\right)$. Then $U \circledast V \in \mathcal{P}^{\prime}\left(\mathcal{S}^{\prime}\right)$ is well defined, moreover $F_{\mathcal{P}}^{\prime \otimes}[U \circledast V]=F_{\mathcal{P}}^{\prime \otimes}[U] \odot F_{\mathcal{P}}^{\prime \otimes}[V]$.

Remind, that spaces $\Gamma(\mathcal{S})$ and $\Gamma\left(\mathcal{S}^{\prime}\right)$ are topological algebras wit respect to operations (6). Polynomial generalization of the Fourier transformation acts as a homomorphism on these algebras. Namely, the following assertion is valid.

Theorem 7. The mappings $F^{\otimes}$ and $F^{\prime \otimes}$ are homomorphisms on algebras $\{\Gamma(\mathcal{S}), \diamond\}$ and $\left\{\Gamma\left(\mathcal{S}^{\prime}\right), \diamond\right\}$ respectively, i.e.

$$
\begin{aligned}
F^{\otimes}[p \diamond q] & =F^{\otimes} p \diamond F^{\otimes} q, \quad \forall p, q \in \Gamma(\mathcal{S}), \\
F^{\prime \otimes}[u \diamond v] & =F^{\prime \otimes} u \diamond F^{\prime \otimes} v, \quad \forall u, v \in \Gamma\left(\mathcal{S}^{\prime}\right) .
\end{aligned}
$$

Proof. The following equalities

$$
\begin{aligned}
F^{\otimes}[p \diamond q]=F^{\otimes}\left[\bigoplus_{n \in \mathbb{Z}_{+}} \sum_{k=0}^{n} \varphi^{\otimes k} \widehat{\otimes} \psi^{\otimes(n-k)}\right]=\bigoplus_{n \in \mathbb{Z}_{+}} F^{\otimes n}\left[\sum_{k=0}^{n} \varphi^{\otimes k} \widehat{\otimes} \psi^{\otimes(n-k)}\right]= \\
=\bigoplus_{n \in \mathbb{Z}_{+}} \sum_{k=0}^{n}(F \varphi)^{\otimes k} \widehat{\otimes}(F \psi)^{\otimes(n-k)}=F^{\otimes} p \diamond F^{\otimes} q, \\
F^{\prime \otimes}[u \diamond v]=F^{\prime \otimes}\left[\underset{n \in \mathbb{Z}_{+}}{\times} \sum_{k=0}^{n} f^{\otimes k} \widehat{\otimes} g^{\otimes(n-k)}\right]=\underset{n \in \mathbb{Z}_{+}}{\times} F^{\otimes n}\left[\sum_{k=0}^{n} f^{\otimes k} \widehat{\otimes} g^{\otimes(n-k)}\right]= \\
=\underset{n \in \mathbb{Z}_{+}}{\times} \sum_{k=0}^{n}\left(F^{\prime} f\right)^{\otimes k} \widehat{\otimes}\left(F^{\prime} g\right)^{\otimes(n-k)}=F^{\otimes} u \diamond F^{\otimes} v,
\end{aligned}
$$

are valid for any $p=\left(\varphi^{\otimes n}\right) \in \Gamma(\mathcal{S}), q=\left(\psi^{\otimes n}\right) \in \Gamma(\mathcal{S}), u=\left(f^{\otimes n}\right) \in \Gamma\left(\mathcal{S}^{\prime}\right), v=\left(g^{\otimes n}\right) \in$ $\Gamma\left(\mathcal{S}^{\prime}\right)$, where $\varphi, \psi \in \mathcal{S}, f, g \in \mathcal{S}^{\prime}$. 
Using the formula (5) following assertion can be proved analogically as the Theorem 7 .

Corollary 4. The mappings $F_{\mathcal{P}}^{\otimes}$ and $F_{\mathcal{P}}^{\prime \otimes}$ are homomorphisms on algebras $\left\{\mathcal{P}\left(\mathcal{S}^{\prime}\right), \cdot\right\}$ and $\left\{\mathcal{P}^{\prime}\left(\mathcal{S}^{\prime}\right), \cdot\right\}$ respectively, i.e.

$$
\begin{aligned}
F_{\mathcal{P}}^{\otimes}[P \cdot Q] & =F_{\mathcal{P}}^{\otimes} P \cdot F_{\mathcal{P}}^{\otimes} Q, \quad \forall P, Q \in \mathcal{P}\left(\mathcal{S}^{\prime}\right), \\
F_{\mathcal{P}}^{\prime \otimes}[U \cdot V] & =F_{\mathcal{P}}^{\prime \otimes} U \cdot F_{\mathcal{P}}^{\prime \otimes} V, \quad \forall U, V \in \mathcal{P}^{\prime}\left(\mathcal{S}^{\prime}\right) .
\end{aligned}
$$

\section{REFERENCES}

1. H.J. Borchers, Algebras of unbounded operators in quantum field theory, Phys. A, 124, №1-3 (1984), 127-144. doi: 10.1016/0378-4371(84)90232-2

2. Yu.A. Brychkov, A.P. Prudnikov, Integral transforms of generalized functions, J. Soviet Math., 34, №3 (1986), 1630-1655.

3. R. Buckdahn, D. Nualart, Linear stochastic differential equations and Wick products, Probab. Theory Relat. Fields, 99 (1994), 501-526.

4. S. Dineen, Complex Analysis on Infinite Dimensional Spaces, Springer-Verlag, London, 1999.

5. K. Grasela, Generalized derivations and Fourier transform of polynomial ultradistributions, Mat. Stud., 20 (2003), №2, 167-178.

6. T. Hida, H.H. Kuo, J. Potthoff, L. Streit, White noise: an infinite dimensional calculus, Kluwer Academic Publ., Dordrecht, 1993.

7. N.A. Kachanovsky, V.A. Tesko, Stochastic integral of Hitsuda-Skorokhod type on the extended Fock space, Ukrainian Math. J., 61 (2009), №6, 873-907.

8. Y.G. Kondratiev, L. Streit, W. Westerkamp, J. Yan, Generalized functions in infinite dimensional analysis, Hiroshima Math. J., 28 (1998), 213-260.

9. O. Lopushansky, S. Sharyn, Polynomial ultradistributions on cone $\mathbb{R}_{+}^{d}$, Topology, 48 (2009), №2-4, 80-90.

10. N. Obata, White noise calculus and Fock space, Springer, 1995.

11. R.S. Pathak, Integral transforms of generalized functions and their applications, Routledge, London, 2017.

12. H. Schaefer, Topological vector spaces, Springer-Verlag, New-York, 1971.

13. S. Sharyn, Application of the functional calculus to solving of infinite dimensional heat equation, Carpathian Math. Publ., 8 (2016), №2, 313-332. doi: 10.15330/cmp.8.2.313-332

14. S. Sharyn, Joint functional calculus in algebra of polynomial tempered distributions, Methods Funct. Anal. Topology, 22 (2016), №1, 62-73.

15. S. Sharyn, Paley-Wiener-type theorem for polynomial ultradifferentiable functions, Carpathian Math. Publ., 7 (2015), №2, 271-279. doi: 10.15330/cmp.7.2.271-279

16. V. Vladimirov, Generalized Functions in Mathematical Physics, Mir, Moscow, 1979.

17. V. Zharinov, Compact families of locally convex topological vector spaces, Fréchet-Schwartz and Dual Fréchet-Schwartz Spaces, Russ. Math. Surv., 34, №4 (1979), 105-143.

Vasyl Stefanyk Precarpathian National University, Ukraine serhii.sharyn@pnu.edu.ua 